\title{
Safety and pharmacokinetic profile of pretomanid (PA-824) in healthy Chinese participants: Results of a phase I single dose escalation study
}

Yue Liu

Clinical trial center, Beijing hospital, National center of gerontology; Institute of geriatric medicine, Chinese academy of medical sciences, Assessment of Clinical Drugs Risk and Individual Application Key Laboratory, Beijing, China.

Yan Tan

Global R\&D center, Shanghai Fosun Pharmaceutical Development, Co., Ltd, Shanghai, China

Gang Wei

Global R\&D center, Shanghai Fosun Pharmaceutical Development, Co., Ltd, Shanghai, China.

Zhifei Lu

Global R\&D center, Shanghai Fosun Pharmaceutical Development, Co., Ltd, Shanghai, China.

Yazhou Liu

Shenyang Hongqi Pharmaceutical Co., Ltd. Shenyang, China

Bo Yang

Shenyang Hongqi Pharmaceutical Co., Ltd. Shenyang, China

Ai-Min Hui ( $\nabla$ aimin.hui@fosunpharma.com )

Global R\&D center, Shanghai Fosun Pharmaceutical Development, Co., Ltd, Shanghai, China.

Kexin Li

Clinical trial center, Beijing hospital, National center of gerontology; Institute of geriatric medicine, Chinese academy of medical sciences, Assessment of Clinical Drugs Risk and Individual Application Key Laboratory, Beijing, China

\section{Research Article}

Keywords: Pretomanid, Tuberculosis, Pharmacokinetics, Safety

Posted Date: December 10th, 2021

DOI: https://doi.org/10.21203/rs.3.rs-1155032/v1

License: (-) (1) This work is licensed under a Creative Commons Attribution 4.0 International License. Read Full License 


\section{Abstract}

We investigated the safety, tolerability and pharmacokinetic (PK) profile of pretomanid (formerly PA-824) in healthy Chinese volunteers. This was a singlecenter, double-blind, placebo-controlled, phase I dose escalation study, in which healthy volunteers were consecutively allocated to increasing pretomanid dose groups $(50,100,200,400,600,800$, or $1000 \mathrm{mg})$ and randomized to receive pretomanid or matching placebo. The primary objective was to evaluate the safety, tolerability and PK profile of pretomanid. In total, 306 volunteers were screened, and 60 were assigned to treatment (pretomanid: $n=46$, placebo: $n=14$ ) of whom $83.3 \%$ were male, age ranged from $19-39$ years and BMI ranged from $19.2-25.9 \mathrm{~kg} / \mathrm{m}^{2}$. At least one adverse event (AE) was reported by $67.4 \%$ of patients assigned to pretomanid and $50.0 \%$ of those who received placebo, there were no serious AEs or AEs leading to withdrawal. Drug-related events that occurred in $\geq 5 \%$ of participants assigned to pretomanid were proteinuria ( $26.1 \%)$, hematuria (15.2\%), conjugated hyperbilirubinemia (6.5\%),

hyperbilirubinemia (6.5\%) and elevated uric acid (6.5\%). No relationship between pretomanid dose and AEs was observed. In the PK analysis ( $\mathrm{n}=46)$, maximum pretomanid plasma concentration was reached in a mean of 4 hours in all dose groups except 800 mg (12 hours) and the plasma half-life ranged from 20.2-25.2 hours. No dose proportionality was observed for maximum plasma concentration, or area under the plasma concentration curve. In conclusion, single pretomanid doses from $50-1000$ mg were well tolerated in healthy Chinese participants and the PK profile was generally consistent with findings in non-Chinese populations.

\section{Introduction}

Tuberculosis (TB) represents a serious global health problem and is one of the leading causes of death worldwide.[1] An estimated 1.5 million people died from TB in 2020. Furthermore, the incidence of TB increased in 2020 due to the COVID-19 pandemic and is forecast to continue to increase into 2022.[1] Around $85 \%$ of people with TB can be successfully treated using combinations of drugs, usually consisting of a core drug with high bactericidal and sterilizing activity and one or more companion drugs used to reduce the risk of treatment failure due to acquisition of resistance to the core drug.[2] However, a significant proportion of patients will develop drug resistant TB, which is difficult to treat.[1] Other current challenges in the treatment of TB include the long duration of current treatment regimens (up to 6 months) and toxicity associated with combination treatment.[3] Long treatment duration and toxicity lead to poor patient compliance, which is a particular challenge and often leads to poor outcomes and increases the risk of developing treatment resistance.

There is therefore a need for novel TB treatment strategies that permit a shorter treatment duration and/or intermittent treatment with wider dosing intervals, and drugs that can successfully treat multi-drug resistant (MDR)-TB. Pretomanid (formerly PA-824) is a compound in the nitroimidazole class that has shown bactericidal and sterilizing activity against Mycobacterium tuberculosis in vitro and in vivo.[4-6] Pretomanid was approved in 2019 by the United States Food and Drug Administration and in 2020 by the European Medicines Agency as part of a multi-drug regimen (with bedaquiline and linezolid [BPaL]) and is recommended by the World Health Organization (WHO) to treat extensively-resistant (XR-TB) and MDR-TB. Pretomanid was also approved in 2016 by the China National Medical Products Administration (NMPA) for use in clinical trials.

The safety and pharmacokinetic (PK) profile of pretomanid has been investigated in multiple phase I and II trials conducted in predominantly Western patients.[7] Results from a total of eight phase I trials of single and repeated administration of pretomanid have been published (CL-001, CL-002, CL-003, CL005, CL-006, CL-009, A5306 and DMID 10-0058), including a total of 289 healthy participants.[8-13] These previous studies investigated single doses of pretomanid ranging from 50 to $1500 \mathrm{mg}$ and repeated doses ranging from 200 to $1000 \mathrm{mg} / \mathrm{day}$ from 7 to 14 days. Furthermore, the PK profile of pretomanid was also investigated in two phase lla studies (CL-007 and CL-010) of pretomanid conducted in tuberculosis patients.[14, 15] The efficacy and safety of pretomanid-containing regimens has been investigated in patients with TB in four phase II studies [16-19] and also in two Phase III studies.[20, 21] A recent systematic review of accumulated clinical data concluded that pretomanid-containing regiment have efficacy in the treatment of rifampicin-resistant and highly resistant TB.[22]

Based on the studies summarized above, the PK profile and safety of pretomanid and pretomanid-containing regimens has been well described in predominantly Western populations of healthy volunteers and patients with TB. However, there have been no studies investigating the PK profile and safety of pretomanid in Chinese patients. The objective of this study was therefore to evaluate the safety, tolerability and PK profile of pretomanid in healthy Chinese volunteers and compare the safety and PK profiles against published data acquired in predominantly Western patient populations.

\section{Materials And Methods Study design and participants}

In this single-center, double-blind, placebo-controlled, phase I dose escalation study (LSF-157-SD), healthy individuals were randomized to receive a single dose of pretomanid (50-1000 mg) or matching placebo. The study protocol was approved by Beijing Hospital Ethics Review Board on October 11, 2017 and was conducted according to the principles of the Declaration of Helsinki Good Clinical Practice, "Technical Guidelines for Clinical Pharmacokinetic Research of Chemical Drugs" and "Guidelines for the Management of Phase I Clinical Trials of Drugs (trial edition)". All patients provided written, informed consent before inclusion. The study was prospectively registered at http://www.chinadrugtrials.org.cn/clinicaltrials.searchlistdetail.dhtml (CTR20181897).

Eligible participants were healthy (based on medical history, physical examination, electrocardiogram, laboratory and other examination results) adults (1940 years) with a body mass index (BMI) of $19-26 \mathrm{~kg} / \mathrm{m}^{2}$, a minimum bodyweight of $55 \mathrm{~kg}$ for males and $50 \mathrm{~kg}$ for females, and a negative urine drug test. Only males were eligible for the 50 and $1000 \mathrm{mg}$ dose cohorts. Female participants were required to provide a negative pregnancy test before inclusion and all participants were required to agree to use effective contraceptive measures for 3 months before signing informed consent until 6 months after study drug administration. Key exclusion criteria included prior receipt of pretomanid, any history of serious illness or other condition that may affect the metabolism or safety of the study drug, positivity for HBV, HCV, HIV or treponema pallidum antibodies, history of peptic ulcers, gastritis, esophagitis or gastroesophageal 
reflux, any history of cardiac abnormalities, hypertension or hypotension, abnormal body temperature, history of hypokalemia or hypomagnesemia, cardiac abnormalities, history of alcohol or drug abuse, use of alcohol within 72 hours of receiving the study drug, history of allergies, use of prescription drugs within 14 days of study drug administration, any history of cardiac abnormalities, use of a potent inhibitor or inducer of cytochrome P450 enzymes, or any drug or substance known to prolong the QT interval within 30 days before study drug administration, ingestion of any product containing grapefruit within 10 days before drug administration, use of any health products, including dietary supplements, within 7 days before drug administration, donation of whole blood or platelets within 56 days before drug administration, participation in other clinical trials within 3 months before screening and pregnant or breast feeding women.

\section{Study procedures}

Pretomanid was provided in tablets of 100 or $200 \mathrm{mg}$ and placebo was provided as identical tablets. Participants were required to fast overnight for at least 10 hours before dosing and received the allocated single oral dose of pretomanid or placebo with $240 \mathrm{~mL}$ of drinking water at around 8:30 am. Patients assigned to higher dose levels were permitted to take the tablets 2 to 3 at a time.

Eligible participants were consecutively allocated to increasing pretomanid dose groups $(50,100,200,400,600,800$, or 1000 mg) and randomized to receive pretomanid or matching placebo. Planned enrolment was 62 patients including 48 patients allocated to pretomanid: $50 \mathrm{mg}(\mathrm{n}=4), 100 \mathrm{mg}(\mathrm{n}=10), 200 \mathrm{mg}$ $(n=10), 400 \mathrm{mg}(n=8), 600 \mathrm{mg}(n=6), 800 \mathrm{mg}(n=6)$, or $1000 \mathrm{mg}(n=4)$, and two patients assigned to placebo at each dose level. Only when the previous dose group was shown to be safe and well-tolerated over a $\geq 7$-day observation period was enrollment to the next dose group initiated. The dosages could be adjusted according to safety and tolerability. The $50 \mathrm{mg}$ starting dose was selected based on the results of international phase I and II clinical studies and the toxicity and safety characteristics reported by pre-clinical studies. $[8-16,18]$ The $100 \mathrm{mg}$ and $200 \mathrm{mg}$ dose levels were chosen to match the doses utilized in international phase III trials of pretomanid,[20,21] and subsequent dose intervals of $200 \mathrm{mg}$ were selected to align with previous phase I studies. The maximum dose of $1000 \mathrm{mg}$ was selected based on previous studies in non-Chinese patients showing that pretomanid absorption reached saturation at this dose level.[9]

\section{Randomization and masking}

Randomized allocation to pretomanid or placebo at each dose level was implemented using a block grouping scheme. Random numbers and the corresponding treatment groups were generated using SAS software 9.4. The randomization table was sealed, in duplicate, and kept by the sponsor and the clinical research unit. Treatment allocation was masked from the participants, investigators and the study sponsor.

\section{Objectives and measurements}

The primary study objective of this study was to evaluate the safety, tolerability and PK profile of a single dose of pretomanid in healthy Chinese individuals.

After providing written, informed consent, participants underwent a full medical examination including collection of demographic information and medical history, measurement of height and bodyweight, physical examination, evaluation of vital signs, laboratory evaluation (blood biochemistry, urinalysis, testing for HBV, HCV, HIV and blood coagulation function), 12-lead ECG, chest X-ray, breath testing for alcohol use and urine testing for recreational drug use and pregnancy test. Participants screened $>7$ days before drug administration underwent a further physical examination, measurement of vital signs, laboratory evaluation, 12-lead ECG, breath testing for alcohol use and urine testing for recreational drug use and pregnancy test on the day before administration of study drug. Following drug administration, participants were monitored in hospital for three days, during which they underwent ECG examination, evaluation of safety and measurement of blood pressure and heart rate at 2, 4, 6, 12, 24, 36 and 48 hours. Safety was further evaluated on Days 4 (72 hours), 5 ( 96 hours) and 6 (120 hours). Respiratory rate was measured 48 hours after administration and a laboratory examination was performed on Day 3 . On Day 6 , all participants underwent physical examination, measurement of vital signs, laboratory evaluation, 12-lead ECG, breath testing for alcohol use and pregnancy test. The QT interval was corrected using the Bazett and Fridericia correction method. Adverse events were categorized and graded based on the Medical Dictionary for Regulatory Activities (MedDRA) v21.0, system organ class (SOC), preferred terminology (PT) and severity.

Samples of around $4 \mathrm{~mL}$ whole blood for PK analysis were obtained one hour before drug administration and following administration at $1,2,4,6,8,12,16$, $24,36,48,72,96$ and 120 hours by direct venipuncture or indwelling catheter in the forearm. The allowable time deviation between the actual PK sampling time was $\pm 2, \pm 5, \pm 10$ and \pm 60 mins for samples obtained at 0-4, 4-8, 8-24 and $>24$ hours, respectively. Blood was collected into an EDTA-K2 (MANUFACTURER) anticoagulated vacuum blood collection tube, and gently inverted several times to ensure mixing with the anticoagulant. After collection, the samples were centrifuged at $2000 \mathrm{~g}$ for 15 minutes at $4^{\circ} \mathrm{C}$. Separated plasma was transferred to screw cap cryotubes and stored at $\leq-70^{\circ} \mathrm{C}$. The total time for sample processing from collection to freezing was controlled at $<60$ minutes. PK samples were sent to a central laboratory for analysis.

\section{Pharmacokinetic analysis}

PK analysis was conducted in a PK analysis set including all subjects who were randomized and received pretomanid with at least one PK parameter measured. The analysis of PK parameters excluded participants in serious violation of the protocol considered to effect the PK analysis or those whose parameters could not be estimated, those whose pre-administration pretomanid concentration was $>5 \%$ of maximum plasma concentration $\left(\mathrm{C}_{\text {max }}\right)$, those who received concomitant medication considered to potentially influence PK parameters, subjects who experienced vomiting within $2 x$ the median time to maximum plasma concentration ( $T_{\text {max }}$ ) of pretomanid, participants for whom the first sample plasma concentration was $C_{\text {max }}$, and those missing sample collections 5-15 after administration.

Plasma concentration of pretomanid was evaluated using liquid chromatography-tandem mass spectrometry (LC MS/MS). The linear range of detection was $20-500 \mathrm{ng} / \mathrm{mL}$, and the lower limit of quantification (LLOQ) was $20 \mathrm{ng} / \mathrm{mL}$. A non-compartmental model was used to calculate parameters including $\mathrm{C}_{\text {max }}$, area under the plasma concentration curve (AUC), $T_{\text {max }}$, terminal elimination half-life $\left(\mathrm{t}_{1 / 2 z}\right)$ and the terminal rate constant $(\lambda z)$. PK parameters were 
summarized using geometric mean, standard deviation (SD) and coefficient of variation (CV) unless otherwise stated. All analyses were conducted using Phoenix WinNonlin 8.1 (Certara Companies, USA). No descriptive statistical analysis of AUC from time 0 to infinity (AUC -inf , $\mathrm{t}_{1 / 2 z}$, or $A U C$ extrapolated from time t to infinity as a percentage of total AUC (AUC_\% Extrap) was performed for subjects with AUC_\% time deviation it was calculated according to the actual sampling time. All blood drug concentration values below the LLOQ were treated as "0" before $T_{\text {max }}$ and as missing after $T_{\max }$. A power function model was used to analyze the proportionality of AUC, $C_{\max }$ and dose (50-1000 mg).[23] The dose relationship was considered proportional if the $90 \%$ confidence interval $(\mathrm{Cl})$ for the estimated gradient of the log regression curve $\left(\beta_{1}\right)$ was completely contained within the critical region defined by $\left(1+\ln \left(\theta_{L}\right) / \ln (r) ; 1+\ln \left(\theta_{H}\right) / \ln (r)\right)$ where $r$ is the ratio between the highest and lowest doses and $\theta_{L}$ and $\theta_{H}$ were set at 0.8 and

1.25 , respectively.

\section{Statistics}

Safety analyses were conducted in all randomized subjects who received the study drug and had at least one safety evaluation. Continuous variables were summarized as mean (SD) or median ( $\max , \mathrm{min}$ ), categorical variables were summarized as frequency and percent. Unless otherwise stated, missing values were not included in the calculation of percentages. All analyses were conducted using SAS version 9.4 (SAS Institute, Inc., Cary, North Carolina, USA).

\section{Results}

\section{Patients}

A total of 306 individuals were screened, 60 were randomized to treatment (pretomanid, $n=46$; placebo, $n=14$ ) (Figure 1). All 60 randomized participants were included in the safety analysis population. Reasons for screening failure were not meeting inclusion/meeting exclusion criteria ( $n=215)$, withdrawal of consent $(n=16)$ or other reason $(n=15)$. Only two patients had recorded concomitant drug use after randomization; one patient assigned to pretomanid 200 $\mathrm{mg}$ (metamizole, cephalexin and cefaclor on Day 3) and one assigned to pretomanid $600 \mathrm{mg}$ (paracetamol, pudilan [a Chinese anti-inflammatory medicine] and cefuroxime axetil on Day 18, after PK sampling had been completed). Neither of these uses of concomitant drugs were considered to have influenced the study results.

Of the 60 participants, $83.3 \%(n=50)$ were male, the ages ranged from 19 to 39 years, height ranged from 148 to $183 \mathrm{~cm}$ and BMI ranged from 19.2 to 25.9 $\mathrm{kg} / \mathrm{m}^{2}$. A total of 33 participants were non-smokers, eight were former smokers and 19 were smokers (all $\leq 5$ cigarettes per day). Detailed patient demographics are presented in Table 1.

Table 1

Participant demographics and baseline characteristics

\begin{tabular}{|c|c|c|c|c|c|c|c|c|c|}
\hline \multirow[t]{2}{*}{ Variable } & \multicolumn{7}{|c|}{ Pretomanid dose group, mg } & \multirow{2}{*}{$\begin{array}{l}\text { All pretomanid } \\
(n=60)\end{array}$} & \multirow{2}{*}{$\begin{array}{l}\text { Placebo } \\
(n=14)\end{array}$} \\
\hline & $\begin{array}{l}50 \\
(n=4)\end{array}$ & $\begin{array}{l}100 \\
(n=9)\end{array}$ & $\begin{array}{l}200 \\
(n=10)\end{array}$ & $\begin{array}{l}400 \\
(n=8)\end{array}$ & $\begin{array}{l}600 \\
(n=6)\end{array}$ & $\begin{array}{l}800 \\
(n=6)\end{array}$ & $\begin{array}{l}1000 \\
(n=3)\end{array}$ & & \\
\hline Males, n (\%) & $4(100.0)$ & 7 (77.8) & $9(90.0)$ & 7 (87.5) & $4(66.7)$ & $5(83.3)$ & $3(100.0)$ & $11(78.6)$ & $50(83.3)$ \\
\hline Mean age, years (SD) & $\begin{array}{l}26.3 \\
(5.19)\end{array}$ & $\begin{array}{l}29.3 \\
(6.24)\end{array}$ & $\begin{array}{l}28.5 \\
(5.68)\end{array}$ & $\begin{array}{l}27.4 \\
(6.55)\end{array}$ & $\begin{array}{l}26.7 \\
(3.78)\end{array}$ & $\begin{array}{l}29.3 \\
(6.98)\end{array}$ & $\begin{array}{l}28.7 \\
(8.62)\end{array}$ & $27.9(4.39)$ & $28.1(5.45)$ \\
\hline Mean height, cm (SD) & $\begin{array}{l}173.8 \\
(5.91)\end{array}$ & $\begin{array}{l}166.8 \\
(7.56)\end{array}$ & $\begin{array}{l}167.8 \\
(4.47)\end{array}$ & $\begin{array}{l}173.8 \\
(7.85)\end{array}$ & $\begin{array}{l}165.5 \\
(9.73)\end{array}$ & $\begin{array}{l}169.2 \\
(6.49)\end{array}$ & $\begin{array}{l}170.7 \\
(6.66)\end{array}$ & $166.4(7.31)$ & $\begin{array}{l}168.6 \\
(7.29)\end{array}$ \\
\hline Mean BMI, kg/m² (SD) & $\begin{array}{l}22.80 \\
(0.804)\end{array}$ & $\begin{array}{l}23.69 \\
(1.779)\end{array}$ & $\begin{array}{l}22.17 \\
(2.158)\end{array}$ & $\begin{array}{l}22.21 \\
(2.188)\end{array}$ & $\begin{array}{l}23.15 \\
(2.033)\end{array}$ & $\begin{array}{l}21.47 \\
(1.808)\end{array}$ & $\begin{array}{l}22.60 \\
(2.506)\end{array}$ & $23.11(1.809)$ & $\begin{array}{l}22.71 \\
(1.935)\end{array}$ \\
\hline \multicolumn{10}{|l|}{ Smoking status, n (\%) } \\
\hline Never smoker & $1(25.0)$ & $4(44.4)$ & $3(30.0)$ & $6(75.0)$ & $4(66.7)$ & $3(50.0)$ & $2(66.7)$ & $10(71.4)$ & $33(55.0)$ \\
\hline Smoker ${ }^{a}$ & $2(50.0)$ & $5(55.6)$ & $7(70.0)$ & $2(25.0)$ & $1(16.7)$ & $2(33.3)$ & 0 & 0 & $19(31.7)$ \\
\hline Quit smoking & $1(25.0)$ & 0 & 0 & 0 & $1(16.7)$ & $1(16.7)$ & $1(33.3)$ & $4(28.6)$ & $8(13.3)$ \\
\hline $\begin{array}{l}\text { Prior medical } \\
\text { condition, n (\%) }\end{array}$ & $1(25.0)$ & $1(11.1)$ & $3(30.0)$ & $1(12.5)$ & $1(16.7)$ & $1(16.7)$ & 0 & $6(42.9)$ & $14(23.3)$ \\
\hline
\end{tabular}

\section{Safety}

Of the 60 patients in the analysis, treatment compliance was 100\%. Among the 46 patients who received pretomanid (All pretomanid group), $67.4 \%$ experienced $\geq 1$ treatment-emergent AE compared with $50.0 \%$ of the patients in the placebo group (Table 2 ). There were no serious AEs or AEs leading to withdrawal, and no deaths occurred. AEs occurring in the pretomanid group were all from the system organ classes of investigations (pretomanid: $63.0 \%$; 
placebo: 42.9\%), gastrointestinal disorders (pretomanid: $8.7 \%$; placebo: $14.3 \%$ ), infections and infestations (pretomanid: $6.5 \%$; placebo: $0 \%$ ), metabolism and nutrition disorders (pretomanid: $2.2 \%$; placebo: $0 \%$ ), nervous system disorders (pretomanid: $2.2 \%$; placebo: $0 \%$ ), respiratory, thoracic and mediastinal disorders (pretomanid:2.2\%; placebo: 7.1\%) and general disorders and administration site conditions (pretomanid: $2.2 \%$; placebo: $0 \%$ ). The most frequently ( $\geq 5 \%$ of subjects) reported TEAE by preferred term was proteinuria, $12(26.1 \%)$ subjects in the all-pretomanid group versus $3(21.4 \%)$ in the placebo group. Other adverse events occurring at a rate $\geq 5 \%$ and greater than placebo included hematuria (15.2\%), elevated uric acid (6.5\%), urine leukocytes (6.5\%) and upper respiratory tract infection (6.5\%). There was no obvious relationship between increasing dose of pretomanid and incidence of AEs. 
Summary of treatment-emergent adverse events

\begin{tabular}{|c|c|c|c|c|c|c|c|c|c|}
\hline & \multicolumn{7}{|c|}{ Pretomanid dose group, $\mathrm{mg}$} & \multirow{2}{*}{$\begin{array}{l}\text { All pretomanid } \\
(n=46)\end{array}$} & \multirow{2}{*}{$\begin{array}{l}\text { Placebo } \\
(n=14)\end{array}$} \\
\hline & $\begin{array}{l}50 \\
(n=4)\end{array}$ & $\begin{array}{l}100 \\
(n=9)\end{array}$ & $\begin{array}{l}200 \\
(n=10)\end{array}$ & $\begin{array}{l}400 \\
(n=8)\end{array}$ & $\begin{array}{l}600 \\
(n=6)\end{array}$ & $\begin{array}{l}800 \\
(n=6)\end{array}$ & $\begin{array}{l}1000 \\
(n=3)\end{array}$ & & \\
\hline$\geq 1 \mathrm{AE}, \mathrm{n}(\%)$ [events] & $\begin{array}{l}2 \\
(50.0) \\
{[5]}\end{array}$ & $\begin{array}{l}3 \\
(33.3) \\
{[8]}\end{array}$ & $\begin{array}{l}8(80.0) \\
{[10]}\end{array}$ & $\begin{array}{l}5 \\
(62.5) \\
{[6]}\end{array}$ & $\begin{array}{l}6(100.0) \\
{[17]}\end{array}$ & $\begin{array}{l}5 \\
(83.3) \\
{[8]}\end{array}$ & $\begin{array}{l}2 \\
(66.7) \\
{[3]}\end{array}$ & $31(67.4)$ [57] & $\begin{array}{l}7(50.0) \\
{[10]}\end{array}$ \\
\hline Serious AEs, $n$ & 0 & 0 & 0 & 0 & 0 & 0 & 0 & 0 & 0 \\
\hline AEs leading to withdrawal, $n$ & 0 & 0 & 0 & 0 & 0 & 0 & 0 & 0 & 0 \\
\hline \multicolumn{10}{|l|}{ AEs by SOC and PT $n(\%)$ [events] } \\
\hline Investigations & $\begin{array}{l}2 \\
(50.0) \\
{[5]}\end{array}$ & $\begin{array}{l}3 \\
(33.3) \\
{[5]}\end{array}$ & $\begin{array}{l}7(70.0) \\
{[8]}\end{array}$ & $\begin{array}{l}5 \\
(62.5) \\
{[6]}\end{array}$ & $\begin{array}{l}6(100.0) \\
{[9]}\end{array}$ & $\begin{array}{l}4 \\
(66.7) \\
{[5]}\end{array}$ & $\begin{array}{l}2 \\
(66.7) \\
{[3]}\end{array}$ & $29(63.0)[41]$ & $6(42.9)[7]$ \\
\hline Proteinuria & $\begin{array}{l}1 \\
(25.0) \\
{[1]}\end{array}$ & $\begin{array}{l}2 \\
(22.2) \\
{[2]}\end{array}$ & $\begin{array}{l}2(20.0) \\
{[2]}\end{array}$ & $\begin{array}{l}3 \\
(37.5) \\
{[3]}\end{array}$ & $\begin{array}{l}1(16.7) \\
{[1]}\end{array}$ & $\begin{array}{l}2 \\
(33.3) \\
{[2]}\end{array}$ & $\begin{array}{l}1 \\
(33.3) \\
{[1]}\end{array}$ & $12(26.1) 12$ & $3(21.4)[3]$ \\
\hline Hematuria & 0 & $\begin{array}{l}1 \\
(11.1) \\
{[1]}\end{array}$ & $\begin{array}{l}3(30.0) \\
{[3]}\end{array}$ & $\begin{array}{l}1 \\
(12.5) \\
{[1]}\end{array}$ & 0 & $\begin{array}{l}1 \\
(16.7) \\
{[1]}\end{array}$ & $\begin{array}{l}1 \\
(33.3) \\
{[1]}\end{array}$ & 7 (15.2) [7] & 0 \\
\hline Conjugated hyperbilirubinemia & $\begin{array}{l}1 \\
(25.0) \\
{[1]}\end{array}$ & 0 & 0 & 0 & $\begin{array}{l}1(16.7) \\
{[1]}\end{array}$ & $\begin{array}{l}1 \\
(16.7) \\
{[1]}\end{array}$ & 0 & $3(6.5)[3]$ & $1(7.1)[1]$ \\
\hline Hyperbilirubinemia & $\begin{array}{l}1 \\
(25.0) \\
{[1]}\end{array}$ & 0 & 0 & 0 & $\begin{array}{l}1(16.7) \\
{[1]}\end{array}$ & $\begin{array}{l}1 \\
(16.7) \\
{[1]}\end{array}$ & 0 & $3(6.5)[3]$ & $1(7.1)[1]$ \\
\hline Elevated uric acid & 0 & 0 & 0 & $\begin{array}{l}1 \\
(12.5) \\
{[1]}\end{array}$ & $\begin{array}{l}2(33.3) \\
{[2]}\end{array}$ & 0 & 0 & $3(6.5)[3]$ & 0 \\
\hline White blood cell count decreased & $\begin{array}{l}1 \\
(25.0) \\
{[1]}\end{array}$ & 0 & 0 & 0 & $\begin{array}{l}1(16.7) \\
{[1]}\end{array}$ & 0 & 0 & $2(4.3)[2]$ & 0 \\
\hline Urine leukocytes & 0 & 0 & $\begin{array}{l}2(20.0) \\
{[2]}\end{array}$ & 0 & $\begin{array}{l}1(16.7) \\
{[1]}\end{array}$ & 0 & 0 & $3(6.5)[3]$ & 0 \\
\hline Platelet count decreased & 0 & 0 & 0 & $\begin{array}{l}1 \\
(12.5) \\
{[1]}\end{array}$ & 0 & 0 & 0 & $1(2.2)[1]$ & 0 \\
\hline Prolonged QT interval on ECG & 0 & $\begin{array}{l}1 \\
(11.1) \\
{[1]}\end{array}$ & 0 & 0 & $\begin{array}{l}1(16.7) \\
{[2]}\end{array}$ & 0 & 0 & $2(4.3)[3]$ & $1(7.1)[1]$ \\
\hline Elevated alanine aminotransferase & $\begin{array}{l}1 \\
(25.0) \\
{[1]}\end{array}$ & 0 & 0 & 0 & 0 & 0 & 0 & $1(2.2)[1]$ & 0 \\
\hline $\begin{array}{l}\text { Increased blood creatine } \\
\text { phosphokinase }\end{array}$ & 0 & 0 & $\begin{array}{l}1(10.0) \\
{[1]}\end{array}$ & 0 & 0 & 0 & 0 & $1(2.2)[1]$ & 0 \\
\hline Blood phosphorus decreased & 0 & 0 & 0 & $\begin{array}{l}1 \\
(12.5) \\
{[1]}\end{array}$ & 0 & 0 & 0 & $1(2.2)[1]$ & 0 \\
\hline Fibrinogen decreased & 0 & 0 & 0 & 0 & 0 & 0 & $\begin{array}{l}1 \\
(33.3) \\
{[1]}\end{array}$ & $1(2.2)[1]$ & $1(7.1)[1]$ \\
\hline Gastrointestinal disorders & 0 & $\begin{array}{l}1 \\
(11.1) \\
{[1]}\end{array}$ & 0 & 0 & $\begin{array}{l}1(16.7) \\
{[2]}\end{array}$ & $\begin{array}{l}2 \\
(33.3) \\
{[2]}\end{array}$ & 0 & $4(8.7)[5]$ & $2(14.3)[2]$ \\
\hline Nausea & 0 & 0 & 0 & 0 & $\begin{array}{l}1(16.7) \\
{[1]}\end{array}$ & $\begin{array}{l}1 \\
(16.7) \\
{[1]}\end{array}$ & 0 & $2(4.3)[2]$ & 0 \\
\hline Vomiting & 0 & 0 & 0 & 0 & $\begin{array}{l}1(16.7) \\
{[1]}\end{array}$ & 0 & 0 & $1(2.2)[1]$ & 0 \\
\hline
\end{tabular}

Adverse events were categorized and graded based on the Medical Dictionary for Regulatory Activities (MedDRA) v21.0.

$\mathrm{AE}$, adverse event; ECG, electrocardiogram; PT, preferred term; SOC, system organ class. 


\begin{tabular}{|c|c|c|c|c|c|c|c|c|c|}
\hline & \multicolumn{7}{|c|}{ Pretomanid dose group, $\mathrm{mg}$} & \multirow{2}{*}{$\begin{array}{l}\text { All pretomanid } \\
(n=46)\end{array}$} & \multirow{2}{*}{$\begin{array}{l}\text { Placebo } \\
(n=14)\end{array}$} \\
\hline & $\begin{array}{l}50 \\
(n=4)\end{array}$ & $\begin{array}{l}100 \\
(n=9)\end{array}$ & $\begin{array}{l}200 \\
(n=10)\end{array}$ & $\begin{array}{l}400 \\
(n=8)\end{array}$ & $\begin{array}{l}600 \\
(n=6)\end{array}$ & $\begin{array}{l}800 \\
(n=6)\end{array}$ & $\begin{array}{l}1000 \\
(n=3)\end{array}$ & & \\
\hline Constipation & 0 & $\begin{array}{l}1 \\
(11.1) \\
{[1]}\end{array}$ & 0 & 0 & 0 & 0 & 0 & $1(2.2)[1]$ & 0 \\
\hline Stomach ache & 0 & 0 & 0 & 0 & $\begin{array}{l}1(16.7) \\
{[1]}\end{array}$ & $\begin{array}{l}1 \\
(16.7) \\
{[1]}\end{array}$ & 0 & $2(4.3)[2]$ & 0 \\
\hline Diarrhea & 0 & $\begin{array}{l}1 \\
(11.1) \\
{[1]}\end{array}$ & 0 & 0 & 0 & 0 & 0 & $1(2.2)[1]$ & $1(7.1)[1]$ \\
\hline Irregular bowel movements & 0 & 0 & 0 & 0 & 0 & 0 & 0 & 0 & $1(7.1)[1]$ \\
\hline Infections and infestations & 0 & 0 & $\begin{array}{l}1(10.0) \\
{[1]}\end{array}$ & 0 & $\begin{array}{l}2(33.3) \\
{[2]}\end{array}$ & 0 & 0 & $3(6.5)[3]$ & 0 \\
\hline Upper respiratory tract infection & 0 & 0 & $\begin{array}{l}1(10.0) \\
{[1]}\end{array}$ & 0 & $\begin{array}{l}2(33.3) \\
{[2]}\end{array}$ & 0 & 0 & $3(6.5)[3]$ & 0 \\
\hline Metabolism and nutrition disorders & 0 & $\begin{array}{l}1 \\
(11.1) \\
{[1]}\end{array}$ & 0 & 0 & 0 & 0 & 0 & $1(2.2)[1]$ & 0 \\
\hline Hypertriglyceridemia & 0 & $\begin{array}{l}1 \\
(11.1) \\
{[1]}\end{array}$ & 0 & 0 & 0 & 0 & 0 & $1(2.2)[1]$ & 0 \\
\hline Nervous system disorders & 0 & 0 & 0 & 0 & $\begin{array}{l}1(16.7) \\
{[1]}\end{array}$ & 0 & 0 & $1(2.2)[1]$ & 0 \\
\hline Dizziness & 0 & 0 & 0 & 0 & $\begin{array}{l}1(16.7) \\
{[1]}\end{array}$ & 0 & 0 & $1(2.2)[1]$ & 0 \\
\hline $\begin{array}{l}\text { Respiratory, thoracic and mediastinal } \\
\text { disorders }\end{array}$ & 0 & 0 & $\begin{array}{l}1(10.0) \\
{[1]}\end{array}$ & 0 & 0 & 0 & 0 & $1(2.2)[1]$ & $1(7.1)[1]$ \\
\hline Rhinorrhea & 0 & 0 & $\begin{array}{l}1(10.0) \\
{[1]}\end{array}$ & 0 & 0 & 0 & 0 & $1(2.2)$ [1] & 0 \\
\hline Oropharyngeal pain & 0 & 0 & 0 & 0 & 0 & 0 & 0 & 0 & $1(7.1)[1]$ \\
\hline $\begin{array}{l}\text { General disorders and administration } \\
\text { site conditions }\end{array}$ & 0 & 0 & 0 & 0 & 0 & $\begin{array}{l}1 \\
(16.7) \\
{[1]}\end{array}$ & 0 & $1(2.2)[1]$ & 0 \\
\hline Chest pain & 0 & 0 & 0 & 0 & 0 & $\begin{array}{l}1 \\
\text { (16.7) } \\
{[1]}\end{array}$ & 0 & 1 (2.2) [1] & 0 \\
\hline
\end{tabular}

\section{PK analysis}

All 46 patients who received pretomanid were included in the PK analysis set. The average plasma concentration-time curves for each pretomanid dose group are shown in Figure 2. The average pretomanid plasma concentration increased with increasing dose at each time point. After 36 hours the pretomanid dose concentration in the $1000 \mathrm{mg}$ dose group was slightly lower than the $800 \mathrm{mg}$ group.

One subject assigned to pretomanid $400 \mathrm{mg}$ was not included in the PK parameter analysis because the first sample was taken at $\mathrm{T}_{\text {max }}$. The average PK parameters for each dose group are summarized in Table 3. $\mathrm{C}_{\text {max }}, \mathrm{AUC}_{0-\mathrm{t}}, \mathrm{AUC}_{0-36}$, and $\mathrm{AUC}_{0 \text {-inf }}$ increased with increasing pretomanid dose from 50 to 1000 mg. Mean $T_{\max }$ was 4 hours across all dose groups except $800 \mathrm{mg}$ which was significantly higher (12 hours). The $\mathrm{T}_{1 / 2 z}$ was similar across all doses and ranged from 20.2 to 25.2 hours. Vd/F and CL/F were similar across the dose groups. Neither $C_{\text {max }}, A C_{0-t}$ or $A_{U} C_{0 \text {-inf }}$ showed proportionality with pretomanid dose as the $90 \% \mathrm{Cl}$ for the gradient of the log regression curve for all three PK variables was not contained within the proportionality boundary (Table 4). A comparison of the pretomanid PK data from this study against data from previous studies conducted in predominantly western populations is shown in Figure 3A-F. 
Table 3

Summary of pretomanid pharmacokinetic parameters $(n=45)$

\begin{tabular}{|c|c|c|c|c|c|c|c|c|c|}
\hline $\begin{array}{l}\text { PK } \\
\text { parameters }^{\mathrm{a}}\end{array}$ & $\begin{array}{l}\mathrm{C}_{\max } \\
\mathrm{ng} / \mathrm{mL}\end{array}$ & $\begin{array}{l}\text { Median } \\
\mathrm{T}_{\max } \mathrm{h} \\
\text { (range) }\end{array}$ & $\begin{array}{l}A_{U C} C_{0-120} \\
\text { h.ng/mL }\end{array}$ & $\begin{array}{l}\mathrm{AUC}_{0-36} \\
\text { h.ng/mL }\end{array}$ & $\begin{array}{l}\mathrm{AUC}_{0 \text {-inf, }} \\
\text { h.ng/mL }\end{array}$ & $t_{1 / 2 z,} h$ & $\lambda z, 1 / h$ & $\mathrm{CL} / \mathrm{F}, \mathrm{L} / \mathrm{h}$ & Vd/F, L \\
\hline \multirow[t]{2}{*}{$50 \mathrm{mg}(\mathrm{n}=4)$} & $324 \pm 91.5$ & \multirow[t]{2}{*}{$4(4,8)$} & $10100 \pm 4160$ & $6940 \pm 2050$ & $10900 \pm 4220$ & $22.4 \pm 6.53$ & $0.0327 \pm 0.00811$ & $5.40 \pm 2.81$ & $161 \pm 56.5$ \\
\hline & $(28.2 \%)$ & & $(41.3 \%)$ & $(29.5 \%)$ & $(38.8 \%)$ & $(29.2 \%)$ & $(24.8 \%)$ & $(52.1 \%)$ & $(35.0 \%)$ \\
\hline \multirow{2}{*}{$\begin{array}{l}100 \mathrm{mg} \\
(\mathrm{n}=9)\end{array}$} & $611 \pm 136$ & \multirow{2}{*}{$\begin{array}{l}4 \\
(2,12)\end{array}$} & $20400 \pm 6420$ & \multirow{2}{*}{$\begin{array}{l}13200 \pm 2560 \\
(19.4 \%)\end{array}$} & $21800 \pm 6720$ & $22.7 \pm 6.25$ & $0.0324 \pm 0.00810$ & $5.14 \pm 2.13$ & $155 \pm 25.6$ \\
\hline & $(22.2 \%)$ & & $(31.5 \%)$ & & $(30.9 \%)$ & $(27.5 \%)$ & $(25.0 \%)$ & $(41.4 \%)$ & $(16.4 \%)$ \\
\hline \multirow{2}{*}{$\begin{array}{l}200 \mathrm{mg} \\
(\mathrm{n}=10)\end{array}$} & $1090 \pm 187$ & \multirow[t]{2}{*}{$4(4,8)$} & $38400 \pm 6710$ & \multirow{2}{*}{$\begin{array}{l}25200 \pm 3670 \\
(14.5 \%)\end{array}$} & $39700 \pm 7220$ & $21.7 \pm 4.83$ & $0.0333 \pm 0.00752$ & $5.19 \pm 0.930$ & $160 \pm 32.8$ \\
\hline & $(17.1 \%)$ & & $(17.4 \%)$ & & $(18.2 \%)$ & $(22.2 \%)$ & $(22.6 \%)$ & $(17.9 \%)$ & $(20.6 \%)$ \\
\hline \multirow{2}{*}{$\begin{array}{l}400 \mathrm{mg} \\
(\mathrm{n}=8)\end{array}$} & $1490 \pm 348$ & \multirow[t]{2}{*}{$4(2,8)$} & $56200 \pm 16900$ & \multirow{2}{*}{$\begin{array}{l}36400 \pm 8810 \\
(24.2 \%)\end{array}$} & $58300 \pm 18500$ & $22.4 \pm 4.61$ & $0.0319 \pm 0.00577$ & $7.49 \pm 2.39$ & $233 \pm 48.7$ \\
\hline & $(23.3 \%)$ & & $(30.0 \%)$ & & $(31.8 \%)$ & $(20.5 \%)$ & (18.1\%) & $(31.9 \%)$ & $(20.9 \%)$ \\
\hline \multirow{2}{*}{$\begin{array}{l}600 \mathrm{mg} \\
(\mathrm{n}=6)\end{array}$} & $2370 \pm 482$ & \multirow{2}{*}{$\begin{array}{l}4 \\
(2,12)\end{array}$} & $98800 \pm 12600$ & \multirow{2}{*}{$\begin{array}{l}61600 \pm 7930 \\
(12.9 \%)\end{array}$} & \multirow{2}{*}{$\begin{array}{l}103000 \pm 13800 \\
(13.4 \%)\end{array}$} & \multirow{2}{*}{$\begin{array}{l}25.2 \pm 4.50 \\
(17.8 \%)\end{array}$} & \multirow{2}{*}{$\begin{array}{l}0.0282 \pm 0.00493 \\
(17.5 \%)\end{array}$} & \multirow{2}{*}{$\begin{array}{l}5.93 \pm 0.810 \\
(13.7 \%)\end{array}$} & \multirow{2}{*}{$\begin{array}{l}214 \pm 37.0 \\
(17.3 \%)\end{array}$} \\
\hline & $(20.3 \%)$ & & $(12.8 \%)$ & & & & & & \\
\hline \multirow{2}{*}{$\begin{array}{l}800 \mathrm{mg} \\
(\mathrm{n}=6)\end{array}$} & $2700 \pm 435$ & \multirow{2}{*}{$\begin{array}{l}12 \\
(4,24)\end{array}$} & $119000 \pm 20600$ & \multirow{2}{*}{$\begin{array}{l}72200 \pm 9930 \\
(13.7 \%)\end{array}$} & $122000 \pm 21400$ & $20.2 \pm 2.97$ & $0.0350 \pm 0.00509$ & $6.74 \pm 1.30$ & $195 \pm 40.1$ \\
\hline & $(16.1 \%)$ & & $(17.2 \%)$ & & $(17.5 \%)$ & $(14.8 \%)$ & $(14.5 \%)$ & $(19.4 \%)$ & $(20.6 \%)$ \\
\hline \multirow{2}{*}{$\begin{array}{l}1000 \mathrm{mg} \\
(n=3)\end{array}$} & $3290 \pm 845$ & \multirow[t]{2}{*}{$4(4,8)$} & $126000 \pm 10300$ & \multirow{2}{*}{$\begin{array}{l}83800 \pm 14200 \\
(17.0 \%)\end{array}$} & $129000 \pm 8050$ & $20.6 \pm 5.51$ & $0.0352 \pm 0.00836$ & $7.76 \pm 0.467$ & $232 \pm 73.8$ \\
\hline & $(25.7 \%)$ & & $(8.2 \%)$ & & $(6.2 \%)$ & $(26.8 \%)$ & $(23.8 \%)$ & $(6.0 \%)$ & $(31.8 \%)$ \\
\hline \multicolumn{10}{|c|}{ aAll parameters are summarized as mean \pm standard deviation (SD) (coefficient of variance [CV, \%]) unless stated. } \\
\hline \multicolumn{10}{|c|}{$\begin{array}{l}\mathrm{AUC}_{0-120} \text {, area under the plasma concentration-time curve from time } 0 \text { to } 120 \text { hours; } \mathrm{AUC}_{0-36} \text {, area under the plasma concentration-time curve from time } \\
0 \text { to } 36 \text { hours; } \mathrm{AUC}_{0 \text {-inf, }} \text { area under the plasma concentration-time curve from time } 0 \text { to infinity; } \mathrm{C}_{\text {max }} \text {, maximum plasma drug concentration; } \mathrm{CL} / \mathrm{F} \text {, } \\
\text { apparent total clearance of the drug from plasma after oral administration; } h \text {, hours; } \lambda z \text {, terminal rate constant; } P K \text {, pharmacokinetic; } t_{1 / 2 z} \text {, terminal } \\
\text { elimination half-life; } T_{\text {max }} \text {, time to maximum plasma drug concentration; } V d / F \text {, apparent volume of distribution. }\end{array}$} \\
\hline
\end{tabular}

Table 4

Relationship between AUC, $\mathrm{C}_{\max }$ and dose

\begin{tabular}{|c|c|c|c|}
\hline & Dose range, $\mathrm{mg}$ & Gradient of the log regression curve $(90 \% \mathrm{Cl})$ & Boundary for dose proportionality ${ }^{a}$ \\
\hline Cmax, ng/mL & $50-1000$ & $0.74(0.68,0.80)$ & $0.9255,1.0745$ \\
\hline AUCO- t, h.ng/mL & $50-1000$ & $0.87(0.79,0.95)$ & $0.9255,1.0745$ \\
\hline AUC0-inf, h.ng/mL & $50-1000$ & $0.85(0.78,0.92)$ & $0.9255,1.0745$ \\
\hline \multicolumn{4}{|c|}{$\begin{array}{l}\text { Defined by }\left(1+\ln \left(\theta_{\mathrm{L}}\right) / \ln (r) ; 1+\ln \left(\theta_{H}\right) / \ln (r)\right) \text { where } r \text { is the ratio between the highest and lowest doses and } \theta_{\mathrm{L}} \text { and } \theta_{\mathrm{H}} \text { were set at } 0.8 \text { and } 1.25 \text {, } \\
\text { respectively. }\end{array}$} \\
\hline
\end{tabular}

\section{Discussion}

Clinical evidence suggests that pretomanid can exert early and sustained bactericidal activity in TB, and is a promising treatment for this disease.[22] In the present study, we show that single doses of pretomanid ranging from 50-1000 mg are well tolerated in healthy Chinese individuals in a fasted state; $67.4 \%$ of patients who were randomized to pretomanid experienced $\geq 1 \mathrm{AE}$ compared with $50.0 \%$ of those who received placebo and no serious $\mathrm{AEs}$ were reported. PK analysis showed that maximum pretomanid plasma concentration was reached approximately 4 hours after administration at all dose levels apart from 800 $\mathrm{mg}$, and the median plasma half-life ranged from 20-25 hours. In general, these findings in a Chinese population were comparable to previous findings of Phase I studies in non-Chinese populations.[8-13]

Most of the AEs observed in this trial were reported in previously published studies of pretomanid and, overall, the safety profile of pretomanid in this trial was consistent with previous findings. $[8-16,18]$ In a pooled analysis of TEAEs in non-Chinese subjects who received pretomanid in prior phase 1 studies (single or multiple doses), the most common TEAEs by system organ class were nervous system disorders (37.7\%), gastrointestinal disorders (24.2\%), and investigations (21.8\%).[24] These align with the most frequently reported system organ classes in the present study, although with a different order of incidence and an overall higher incidence rate. In the previous phase 1 studies, the most frequently reported AEs were headache (31.5\%), nausea (11.8\%), 
hemoglobin decreased (10.7\%), and contact dermatitis (11.4\%).[24] In the present study, most of the TEAEs were from the system organ class of investigations, with proteinuria and hematuria the most frequently reported AEs. Furthermore, the incidence rate of proteinuria was comparable between the all pretomanid group and the placebo group. In contrast, the incidence of hematuria was higher for patients receiving pretomanid versus placebo, however, this result should be interpreted cautiously given the small sample size. Headache, contact dermatitis and decreases in hemoglobin were not observed in the Chinese subjects in this study, while nausea was observed in 2 (4.3\%) subjects in the all pretomanid group (versus 0 in the placebo group), which is in contrast to the findings from the previous phase 1 studies.

In general, the PK findings of this study support prior studies in non-Chinese populations. The increase in $\mathrm{C}_{\max }$ with increasing pretomanid dose from 50 $1000 \mathrm{mg}$ observed in the present study is consistent with previous studies in non-Chinese populations and the range of $\mathrm{C}_{\text {max }}$ values is comparable (Figure $3 A)$. $[8-16,18]$ In previous studies, the pretomanid AUC was approximately dose proportional close to the labelled dose of $200 \mathrm{mg}(50-200 \mathrm{mg})$ but became less than proportional at doses from 200-1000 mg.[8-16, 18] In contrast, in the present study $\mathrm{AUC}_{0-\mathrm{t}}$ and $\mathrm{AUC}_{0 \text {-inf }}$ increased with increasing pretomanid dose from 50-1000 mg but were not dose proportional. However, the $\mathrm{AUC}_{0 \text {-inf }}$ results at doses around the approved dose of $200 \mathrm{mg}$ are generally in agreement with previous studies in non-Chinese populations (Figure $3 B$ ). [8-16, 18] it should be noted that $\mathrm{AUC}_{0-\mathrm{t}}$ is very dependent on study methodology, such as exact sampling times, and therefore it is not as meaningful to make cross-study comparisons of this parameter. Our results also showed a median pretomanid $\mathrm{T}_{\max }$ of 4 hours in all dose groups except for the $800 \mathrm{mg}$ dose group, which is similar to previous studies in non-Chinese populations which reported $T_{\max }$ of 4-6.5 hours (Figure $3 \mathrm{C}$ ). Interestingly, the $\mathrm{T}_{\max }$ results of the $800 \mathrm{mg}$ group in the present study are also higher than previous findings at this dose, for example, the median value of $T_{\max }$ in the $800 \mathrm{mg}$ group in CL-005 was 5 hours. Finally, the average values of $\mathrm{t}_{1 / 2 \mathrm{z}} \mathrm{CL}$ and $\mathrm{Vd}$ in the present study were 20.6-25.2 hours, 5.14-7.76 L/h and 155-232 L, respectively compared with 13.5-23.2 hours, 4.6-16.6 L/h and 173-421.65 L in previous studies, respectively (Figure 3D, E and F). Considering that pretomanid is extensively metabolized in the human body and the majority is excreted in the urine in the form of metabolites. Therefore, as expected, the elimination of pretomanid and its distribution in the Chinese subjects included in this analysis is slightly different from previous studies in non-Chinese populations and may be related to differences in factors such as diet, culture, BMI.

The primary limitation of this study was the single-dose design and the healthy volunteer population, which do not allow generalization to multiple doses or use of pretomanid as part of a multi-drug regimen. Further data are required to confirm the efficacy and safety of pretomanid in Chinese populations when given as part of combination treatment.

\section{Conclusions}

The results of this study show that single pretomanid doses from 50-1000 mg are well tolerated in healthy Chinese participants and the safety and PK profiles are generally consistent with those reported in non-Chinese populations.

\section{Declarations}

\section{Acknowledgements}

Editorial support for this manuscript was provided by Jake Burrell PhD (Rude Health Consulting) and paid for by Shanghai Fosun Pharmaceutical Development, Co., Ltd.

We thank Changjiang Xu, Te Li (previous staff of Shanghai Fosun Pharmaceutical Development, Co., Ltd.) for the help of study design.

\section{Funding}

This study was funded by Shanghai Fosun Pharmaceutical Development, Co., Ltd.

\section{Conflict of interest}

Yan Tan, Gang Wei, Zhifei Lu, Ai-Min Hui are the full-time employee of Shanghai Fosun Pharmaceutical Development, Co., Ltd. Yazhou Liu, Bo Yang are the full-time employee of Shenyang Hongqi Pharmaceutical Co., Ltd.

\section{Author contributions}

Yazhou Liu, Bo Yang, Ai-Min Hui, Kexin Li contributed to the study conception and design. Material preparation, data collection and analysis were performed by Yue Liu, Yan Tan, Gang Wei, Zhifei Lu. All authors participated in the data interpretation, draft review and final version approval.

\section{References}

1. World Health Organization. Global tuberculosis report 2021. Geneva: WHO; 2021. Available from: https://www.who.int/teams/global-tuberculosisprogramme/tb-reports.

2. Van Deun A, Decroo T, Piubello A, de Jong BC, Lynen L, Rieder HL. Principles for constructing a tuberculosis treatment regimen: the role and definition of core and companion drugs. Int J Tuberc Lung Dis. 2018;22(3):239-45.

3. Furin J, Cox H, Pai M. Tuberculosis. Lancet. 2019;393(10181):1642-56.

4. Stover CK, Warrener P, VanDevanter DR, Sherman DR, Arain TM, Langhorne MH, et al. A small-molecule nitroimidazopyran drug candidate for the treatment of tuberculosis. Nature. 2000;405(6789):962-6. 
5. Singh R, Manjunatha U, Boshoff HI, Ha YH, Niyomrattanakit P, Ledwidge R, et al. PA-824 kills nonreplicating Mycobacterium tuberculosis by intracellular NO release. Science. 2008;322(5906):1392-5.

6. Tyagi S, Nuermberger E, Yoshimatsu T, Williams K, Rosenthal I, Lounis N, et al. Bactericidal activity of the nitroimidazopyran PA-824 in a murine model of tuberculosis. Antimicrob Agents Chemother. 2005;49(6):2289-93.

7. Stancil SL, Mirzayev F, Abdel-Rahman SM. Profiling Pretomanid as a Therapeutic Option for TB Infection: Evidence to Date. Drug Des Devel Ther. 2021;15:2815-30.

8. Dooley KE, Luetkemeyer AF, Park JG, Allen R, Cramer Y, Murray S, et al. Phase I safety, pharmacokinetics, and pharmacogenetics study of the antituberculosis drug PA-824 with concomitant lopinavir-ritonavir, efavirenz, or rifampin. Antimicrob Agents Chemother. 2014;58(9):5245-52.

9. Ginsberg AM, Laurenzi MW, Rouse DJ, Whitney KD, Spigelman MK. Safety, tolerability, and pharmacokinetics of PA-824 in healthy subjects. Antimicrob Agents Chemother. 2009;53(9):3720-5.

10. Ginsberg AM, Laurenzi MW, Rouse DJ, Whitney KD, Spigelman MK. Assessment of the effects of the nitroimidazo-oxazine PA-824 on renal function in healthy subjects. Antimicrob Agents Chemother. 2009;53(9):3726-33.

11. Li M, Saviolakis GA, El-Amin W, Makhene MK, Osborn B, Nedelman J, et al. Phase 1 Study of the Effects of the Tuberculosis Treatment Pretomanid, Alone and in Combination With Moxifloxacin, on the QTc Interval in Healthy Volunteers. Clin Pharmacol Drug Dev. 2021;10(6):634-46.

12. Winter H, Egizi E, Erondu N, Ginsberg A, Rouse DJ, Severynse-Stevens D, et al. Evaluation of pharmacokinetic interaction between PA-824 and midazolam in healthy adult subjects. Antimicrob Agents Chemother. 2013;57(8):3699-703.

13. Winter H, Ginsberg A, Egizi E, Erondu N, Whitney K, Pauli E, et al. Effect of a high-calorie, high-fat meal on the bioavailability and pharmacokinetics of PA824 in healthy adult subjects. Antimicrob Agents Chemother. 2013;57(11):5516-20.

14. Diacon AH, Dawson R, Hanekom M, Narunsky K, Maritz SJ, Venter A, et al. Early bactericidal activity and pharmacokinetics of PA-824 in smear-positive tuberculosis patients. Antimicrob Agents Chemother. 2010;54(8):3402-7.

15. Diacon AH, Dawson R, du Bois J, Narunsky K, Venter A, Donald PR, et al. Phase II dose-ranging trial of the early bactericidal activity of PA-824. Antimicrob Agents Chemother. 2012;56(6):3027-31.

16. Dawson R, Diacon AH, Everitt D, van Niekerk C, Donald PR, Burger DA, et al. Efficiency and safety of the combination of moxifloxacin, pretomanid (PA824), and pyrazinamide during the first 8 weeks of antituberculosis treatment: a phase $2 \mathrm{~b}$, open-label, partly randomised trial in patients with drugsusceptible or drug-resistant pulmonary tuberculosis. Lancet. 2015;385(9979):1738-47.

17. Diacon AH, Dawson R, von Groote-Bidlingmaier F, Symons G, Venter A, Donald PR, et al. Bactericidal activity of pyrazinamide and clofazimine alone and in combinations with pretomanid and bedaquiline. Am J Respir Crit Care Med. 2015;191(8):943-53.

18. Diacon AH, Dawson R, von Groote-Bidlingmaier F, Symons G, Venter A, Donald PR, et al. 14-day bactericidal activity of PA-824, bedaquiline, pyrazinamide, and moxifloxacin combinations: a randomised trial. Lancet. 2012;380(9846):986-93.

19. Tweed CD, Dawson R, Burger DA, Conradie A, Crook AM, Mendel CM, et al. Bedaquiline, moxifloxacin, pretomanid, and pyrazinamide during the first 8 weeks of treatment of patients with drug-susceptible or drug-resistant pulmonary tuberculosis: a multicentre, open-label, partially randomised, phase $2 \mathrm{~b}$ trial. Lancet Respir Med. 2019;7(12):1048-58.

20. Conradie F, Diacon AH, Ngubane N, Howell P, Everitt D, Crook AM, et al. Treatment of Highly Drug-Resistant Pulmonary Tuberculosis. N Engl J Med. 2020;382(10):893-902.

21. Tweed CD, Wills GH, Crook AM, Amukoye E, Balanag V, Ban AYL, et al. A partially randomised trial of pretomanid, moxifloxacin and pyrazinamide for pulmonary TB. Int J Tuberc Lung Dis. 2021;25(4):305-14.

22. Gils T, Lynen L, de Jong BC, Van Deun A, Decroo T. Pretomanid for tuberculosis: a systematic review. Clin Microbiol Infect. 2021.

23. Smith BP, Vandenhende FR, DeSante KA, Farid NA, Welch PA, Callaghan JT, et al. Confidence interval criteria for assessment of dose proportionality. Pharm Res. 2000;17(10):1278-83.

24. FDA Briefing Document. Pretomanid Tablet, $200 \mathrm{mg}$. Meeting of the Antimicrobial Drugs Advisory Committee (AMDAC). Available from: https://www.fda.gov/media/127592/download. Accessed November 2021.

\section{Figures}




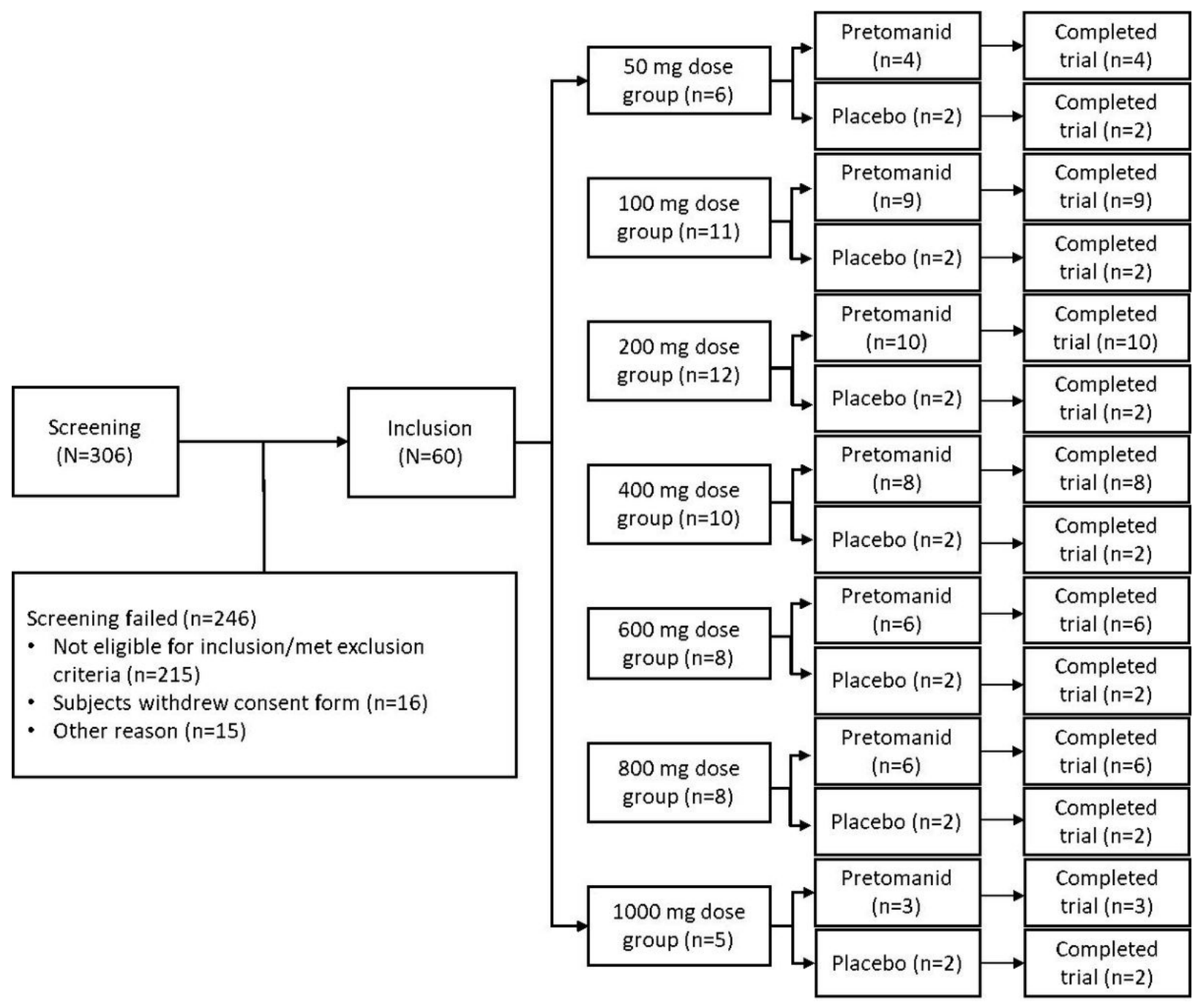

Figure 1

Patient flow diagram 

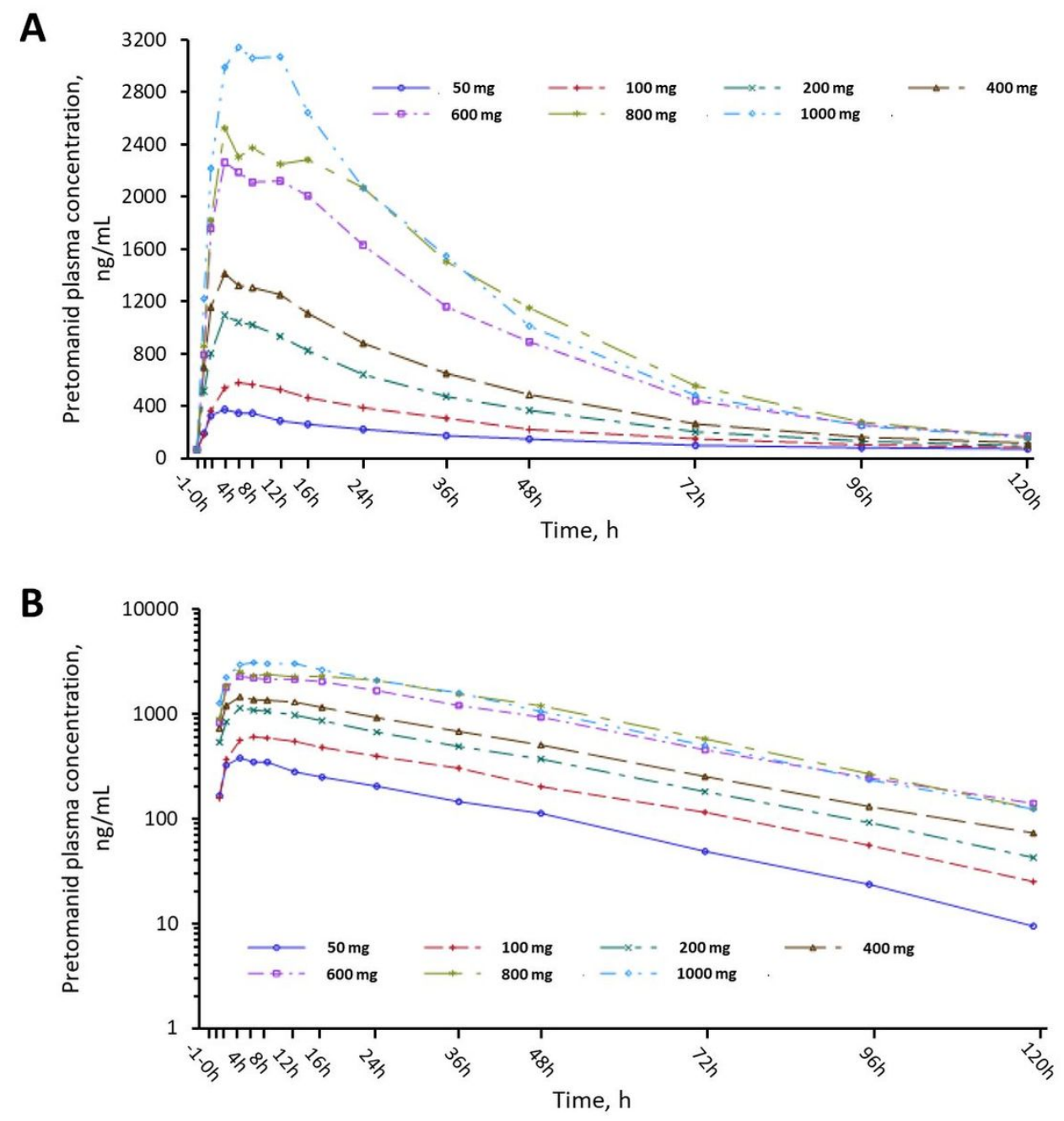

Figure 2

Pretomanid plasma concentration-time curve: A) linear plot, B) semi-log plot ( $n=46)$ 
A

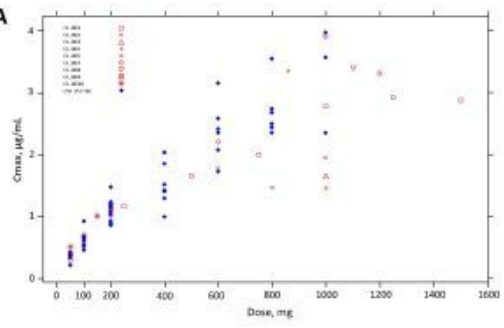

D

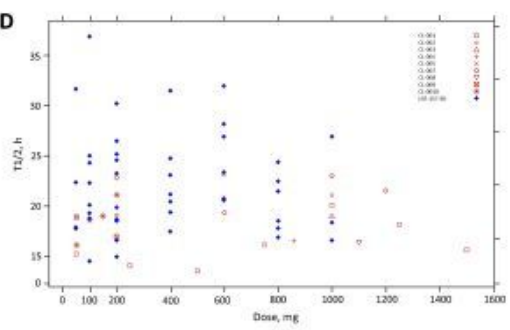

B

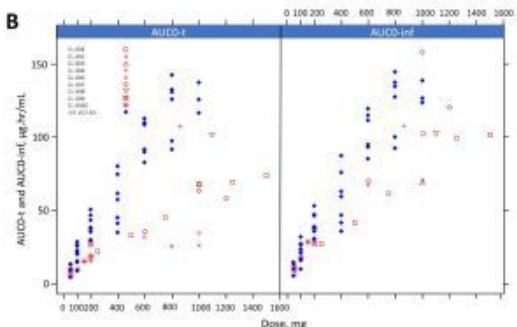

E

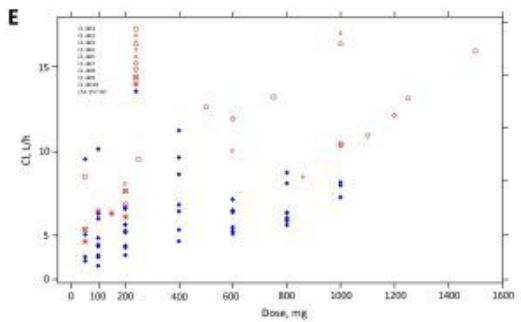

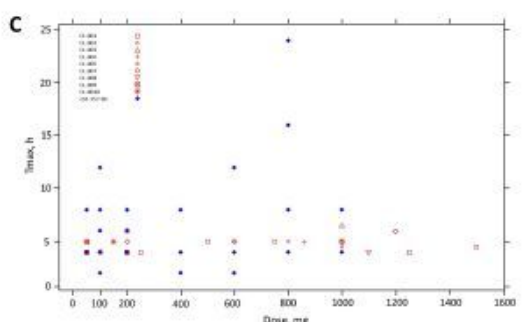

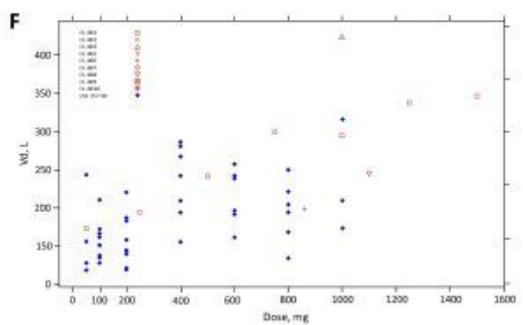

\section{Figure 3}

Comparison of the pharmacokinetic profile of pretomanid in Chinese and non-Chinese subjects: A) Cmax, B) AUC, C) Tmax, D) T1/2z, E) CL, F) Vd Data in non-Chinese subjects were extracted from the following previous studies: CL-001 and CL-002 [9], CL-003 [13], CL-004 (ADME study, data on file[NCT03202693]), CL-005 [10], CL-007 [14], CL-008 (ADME study, data on file), CL-009 [13] and CL-010 [15]. Note: Studies CL-007 and CL-010 were multiple dose studies, however, single dose data were extracted and used to produce these graphs. AUC0-inf, area under the plasma concentration-time curve from time 0 to infinity; AUCO-t, area under the plasma concentration-time curve from time 0 to thours; $\mathrm{Cmax}$, maximum plasma drug concentration; $\mathrm{CL}$, apparent total clearance of the drug from plasma after oral administration; h, hours; t1/2z, terminal elimination half-life; Tmax, time to maximum plasma drug concentration; Vd, apparent volume of distribution. LSF-157-SD is the present analysis. 